\title{
Post-Breast Cancer Radiotherapy Bronchiolitis Obliterans Organizing Pneumonia
}

\author{
Gary R Epler and Eileen M Kelly
}

\begin{abstract}
BACKGROUND: Radiotherapy for breast cancer has been implicated in the development of bronchiolitis obliterans organizing pneumonia (BOOP). Patients may be asymptomatic or may have pulmonary and constitutional symptoms that are moderate or severe. Postradiotherapy BOOP usually develops during the 12 months after completion of radiotherapy and is characterized by ground-glass opacities in the radiation-exposed lung and frequently in the non-irradiated lung. METHODS: An updated literature search and review was performed to update the systematic review we conducted in 2014. Ten new publications were identified: 2 Japanese epidemiological studies, 1 Japanese case series study, 6 case reports, and 1 review article. RESULTS: The incidence of postradiotherapy BOOP was $1.4 \%$ in both Japanese epidemiological studies. Risk factors included increasing age, cigarette smoking, and increasing central lung distance. The case reports included 7 women who had breast cancer postradiation BOOP and 1 woman who had an ataxia telangiectasia mutated $(A T M)$ gene mutation, which may increase radiation sensitivity. CONCLUSION: Postradiotherapy BOOP in women with breast cancer occurs at a rate of 1.0-3.0\% and may occur in women with immune system dysfunction and genetic mutations. Key words: breast cancer; lung cancer; radiation therapy; postradiotherapy; BOOP; ATM gene. [Respir Care 2020;65(5):686-692. (C) 2020 Daedalus Enterprises]
\end{abstract}

\section{Introduction}

Radiation therapy plays a key role in the treatment of breast cancer. Lumpectomy and radiation therapy are the preferable treatments for early-stage breast cancer because they provide survival rates equal to that of total mastectomy and axillary dissection while preserving the breast. $^{2}$ Radiation therapy for breast cancer has traditionally been delivered to the whole breast at a dose of 45-50 Gray with 1.8-2.0 Gray per fraction. ${ }^{2}$ Newer regimens that include hypofractionated whole-breast irradiation, partial-breast irradiation, brachytherapy, and intra-operative radiation

\footnotetext{
Dr Epler is affiliated with the Department of Pulmonary and Critical Care Medicine, Harvard Medical School, Brigham and Women's Hospital, Boston, Massachusetts. Dr Kelly is located in Smithtown, New York.

The authors have disclosed no conflicts of interest.

Correspondence: Gary R Epler MD, Pulmonary and Critical Care Medicine, Harvard Medical School, 15 Francis Street, Brigham and Women's Hospital, Boston, Massachusetts 02115. E-mail: gepler@ bwh.harvard.edu.
}

DOI: $10.4187 /$ respcare. 07150 therapy have been developed, tested, and adopted to varying degrees. ${ }^{1}$

Bronchiolitis obliterans organizing pneumonia (BOOP) is an inflammatory lung disorder that can be caused by both infectious and noninfectious processes. Idiopathic BOOP is also known as cryptogenic organizing pneumonia. $\mathrm{BOOP}$ is defined as organized polypoid granulation tissue in the distal airways extending into the alveolar ducts and alveoli. ${ }^{3}$ Noninfectious causes of BOOP include rheumatologic and connective-tissue diseases, inflammatory bowel disease, reactions from drugs, fumes, and other toxic exposures, and radiation therapy to the chest. ${ }^{4}$ This inflammatory lesion is caused by a cascade of cytokine events that differs from inflammation occurring in asthma, bronchitis, and nonspecific interstitial pneumonia because there is no fibrosing process. ${ }^{4}$ Patients with BOOP often present with fever, cough, shortness of breath, and fatigue. Crackles are commonly heard on auscultation. Unilateral or bilateral patchy infiltrates are noted on chest radiographs, and chest computed tomography (CT) findings show ground-glass opacities, consolidation, air bronchograms, pleural-based triangle-shaped opacities, and sometimes the reversed halo sign. ${ }^{3,4}$ Pulmonary function tests may show decreased vital capacity and moderate decrease in diffusing capacity with 


\section{Post-Breast CANCER RADIOTHERAPY AND BOOP}

no obstruction of air flow. ${ }^{4}$ Lung biopsies show wellformed plugs of edematous granulation tissue involving terminal and respiratory bronchioles extending into alveolar ducts and alveoli, including polypoid plugs infiltrated by nests of chronic inflammatory cells. ${ }^{3}$ The histology may be characterized by fatty alveolar macrophages and interstitial infiltrates of lymphocytes and plasma cells. Corticosteroid therapy is the primary treatment, and relapses occur among one quarter to one third of patients when corticosteroid dose is tapered or stopped; recurrences typically respond to the same dose of corticosteroid. Up to $80 \%$ of patients with BOOP are cured, and mortality has been estimated to be about $5 \%{ }^{4,5}$

The first reports of BOOP occurring in patients receiving radiotherapy for breast cancer appeared in $1995 .{ }^{6,7}$ In 2014 , a systematic literature review of postradiotherapy BOOP in women with breast cancer identified 38 articles that contained clinical information from 199 subjects. ${ }^{1}$ The epidemiological studies were reported from Japan, and the occurrence ranged from $0.8 \%$ to $2.9 \%$. Nonproductive cough and fever were the most common symptoms. Contralateral involvement of BOOP occurred in 8-100\% of subjects. Most but not all subjects were treated with corticosteroids. Relapses occurred in some subjects, and there were no deaths. The largest case series was from France and consisted of 15 women who developed BOOP after breast radiotherapy. ${ }^{8}$ Pulmonary symptoms occurred 3-47 weeks after radiation treatment. The contralateral lung was involved in $47 \%$ of patients. All 15 patients were treated with corticosteroids, and there was a high relapse rate of $80 \%$. There were 24 case reports from a number of different countries. Most patients were originally diagnosed with community-acquired pneumonia, and one third also had radiation pneumonitis. One third of patients were hospitalized, and BOOP occurred in the contralateral lung in $60 \%$ patients, usually at initial diagnosis but also at relapse. Among these patients, $70 \%$ were treated with corticosteroids, from as little as 1 dose to treatment lasting for a year or longer. Among them, $65 \%$ had a relapse and $15 \%$ had $>1$ relapse. ${ }^{1}$

\section{Methods}

We sought to update the 2014 review and to explore new risk factors as well as genetic and immunological implications. A PubMed literature search was conducted using key words breast cancer, lung cancer, radiation, radiotherapy, BOOP, organizing, pneumonia, cryptogenic, and bronchiolitis. Letters to the editor and correspondence were included in the search. Reports that were not available in English were not included.

Ten relevant publications were identified in the search. Of the 10 reports, 7 were from Japan, and 3 were from Europe. Two were Japanese retrospective epidemiological
Table 1. Demographic, Clinical, and Treatment Characteristics From Japanese Epidemiological Studies and Case Series

\begin{tabular}{|c|c|c|c|}
\hline $\begin{array}{c}\text { Clinical } \\
\text { Characteristics }\end{array}$ & 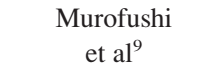 & $\begin{array}{l}\text { Sato } \\
\text { et } \mathrm{al}^{10}\end{array}$ & $\begin{array}{l}\text { Kawakami } \\
\text { et } \mathrm{al}^{12}\end{array}$ \\
\hline Study & $\begin{array}{l}\text { Epidemiological } \\
\text { study }\end{array}$ & $\begin{array}{l}\text { Epidemiological } \\
\text { study }\end{array}$ & Case series \\
\hline Total & 1,176 & 665 & NA \\
\hline BOOP & 16 & 9 & 8 \\
\hline BOOP, & 1.4 & 1.4 & NA \\
\hline Median age, y & 57 & 52 & 56.8 (mean) \\
\hline $\begin{array}{l}\text { Median symptom } \\
\text { onset latency, mo }\end{array}$ & 4.9 & 2-12 (range) & 5.6 \\
\hline Fever & $7(44)$ & $8(89)$ & $7(88)$ \\
\hline Cough & $16(100)$ & $7(77)$ & $7(88)$ \\
\hline Dyspnea & $1(6)$ & 0 & 0 \\
\hline Antibiotic treatment & $12(75)$ & ND & $5(62)$ \\
\hline $\begin{array}{l}\text { Corticosteroid } \\
\text { treatment }\end{array}$ & $2(13)$ & $9(100)$ & $3(38)$ \\
\hline $\begin{array}{l}\text { Relapse after } \\
\text { corticosteroid } \\
\text { discontinuation }\end{array}$ & $2(100)$ & $2(22)$ & $3(100)$ \\
\hline \multicolumn{4}{|c|}{$\begin{array}{l}\text { Data are presented as } n \text { or } n(\%) \text {. } \\
\text { BOOP }=\text { bronchiolitis obliterans organizing pneumonia } \\
\text { NA }=\text { not applicable } \\
\text { ND }=\text { no data }\end{array}$} \\
\hline
\end{tabular}

studies, 1 report was a Japanese case series study, 6 papers were individual case reports, and 1 result was a review article. There were no new reports from the United States.

\section{Results}

\section{Retrospective Epidemiological Analyses}

The demographic, clinical, and treatment information from the 2 epidemiological studies and case series are presented in Table 1 . Murofushi et $\mathrm{al}^{9}$ retrospectively analyzed data from 1,176 subjects with breast cancer who received radiation therapy after breast-conserving surgery or after mastectomy at the Cancer Institute of the Japanese Foundation. They reported BOOP in 16 subjects (1.4\%); 12 subjects had breast-conserving surgery before radiotherapy, and 4 had radiotherapy after mastectomy. The median follow-up period was 25.5 months, with a range of 8.1-53.4 months.

Subjects at this institution had chest radiographs every 3-6 months after treatment or if they developed respiratory symptoms. The median time after completing radiation therapy to diagnosis of BOOP was 4.9 months. All 16 subjects reported cough, 7 had fever, 4 had fatigue, and 1 subject had decreased oxygen saturation. Symptoms and radiographic abnormalities resolved in 12 subjects who received antibiotics and antitussives, while 2 subjects 


\section{Post-Breast CANCER RADIOTHERAPY AND BOOP}

were treated with corticosteroid therapy. Both of these subjects had a relapse that required a second course of corticosteroid treatment.

In the univariate analysis, age 52 years or greater was significantly associated with $\mathrm{BOOP}$ for 16 subjects $(P=$ $.02)$ and for the 12 subjects who received breast-conserving therapy $(P=.039)$. Chemotherapy, endocrine therapy, and maximum oxygen uptake were not significant. In the multivariate analysis, increasing age was associated with an increased incidence of BOOP, with an odds ratio of 1.58 (95\% CI 1.01-2.28). In this multivariate analysis the incidence of BOOP was higher for smokers, with an odds ratio 3.09 (95\% CI 1.01-9.48) for all subjects and 4.12 (95\% CI $1.12-15.11)$ for the 12 subjects who received breast-conserving therapy. Endocrine therapy and chemotherapy had no effect.

Sato et $\mathrm{al}^{10}$ retrospectively analyzed data from 665 women with breast cancer who received breast-conserving surgery as initial treatment for their breast cancer. The median age was 56 y and $97 \%$ of the subjects were followed up for at least 12 months. Most subjects were treated with whole-breast radiotherapy at a dose of $50 \mathrm{~Gy} / 25$ fractions over 5 weeks. Those with positive surgical margins received a boost of 10 Gy over 5 fractions. Subjects were evaluated for pulmonary toxicity with chest radiographs or chest CT scans at 1, 3, 9, 15, and 21 months after completion of radiotherapy.

Organizing pneumonia developed in 9 subjects (1.4\%). Six of the 9 subjects developed organizing pneumonia within 6 months after radiotherapy (range 2-12 months). All subjects were symptomatic with mild fever and or cough. All 9 subjects were treated with corticosteroids; 2 subjects relapsed and received additional corticosteroid therapy, with 1 subject continuing on low-dose steroid therapy 6 years after initial diagnosis. The average duration of steroid therapy was 9.5 months (range 1-72 months).

Univariate analysis showed that central lung distance was significantly related to development of postradiotherapy organizing pneumonia. Central lung distance is measured from the radiation field border to the edge of the lung contour at the central axis on the tangential simulation digitally reconstructed radiograph, and the volume of lung radiated varies from $4 \%$ to $24 \%$ of the ipsilateral lung depending upon the radiation plan. ${ }^{11}$ In the report by Sato et al, ${ }^{10} 2$ subjects with central lung distance $\leq 1.5 \mathrm{~cm}$ developed organizing pneumonia, and 7 subjects with central lung distance $\geq 1.5$ developed organizing pneumonia $(P=.004)$.

\section{Retrospective Case Series}

Kawakami et $\mathrm{al}^{12}$ reported data from 8 subjects with postradiotherapy breast cancer organizing pneumonia. The average age was $56.8 \mathrm{y}$ and the median duration from completion of therapy to symptoms was 5.6 months. Seven subjects were symptomatic with fever and cough. No patients were hypoxic. Three subjects received corticosteroid treatment, and 5 subjects had none with eventual resolution. Those receiving corticosteroids all experienced a recurrence and had a longer time to chest imaging resolution.

One subject was a 62-y-old woman with right breast cancer who received 50 Gy radiotherapy. Fourteen months af ter radiotherapy completion, she reported fever and cough that did not respond to antibiotics. The chest CT showed consolidation in the right upper, middle, and lower lobes. Bronchoalveolar lavage fluid revealed increased lymphocytes and decreased $\mathrm{CD} 4 / 8$ ratio leading to a diagnosis of radiation-induced organizing pneumonia. Because the subject complained of severe respiratory discomfort on exertion, she received $500 \mathrm{mg}$ methylprednisolone for $3 \mathrm{~d}$ and then $20 \mathrm{mg}$ prednisolone a day. Eight months later, the infiltrates resolved, and the corticosteroid therapy was discontinued. One month later, a new left lung consolidation appeared. She was not hypoxic, so she was monitored with no prednisone treatment, and the infiltrate resolved in 3 months.

\section{Patient Case Studies}

The demographic, clinical, and treatment characteristics from the case reports are shown in Table 2. Jacobs et $\mathrm{al}^{13}$ described a 56-y-old woman with bilateral breast cancer who had surgery, unilateral radiotherapy, and endocrine therapy. After developing hepatic, pulmonary, and skeletal metastases, she was treated with doxorubicin monotherapy. Prior to her fifth cycle of doxorubicin, she developed rapidly worsening breathlessness and became cyanotic. The chest CT scan showed bilateral ground-glass opacities. Transbronchial lung biopsy showed intra-alveolar granulation tissue consistent with BOOP. Treatment with dexamethasone resulted in rapid improvement, and her chest radiograph was normal by her eighth day of dexamethasone. Doxorubicin, radiation therapy, or both may have caused this subject's BOOP.

Bissoli et al ${ }^{14}$ described a 71-y-old woman who had right breast cancer treated with quadrantectomy and radiation therapy. She developed fever, sweats, and nonproductive cough that persisted with antibiotic therapy. Chest radiography revealed right lung consolidation, and the CT scan showed air bronchograms within this infiltrate. A repeat CT scan 3 weeks later showed new ground-glass opacities in the right upper lung. Transbronchial biopsy confirmed BOOP. The patient was treated with prednisone with dramatic resolution of symptoms within 1 week, and the chest CT scan 3 weeks later was normal.

Inoue et $\mathrm{al}^{15}$ described a 66 -y-old woman who reported cough with sputum for 4 weeks that occurred 23 weeks 


\section{Post-Breast CANCER RADIOTHERAPY AND BOOP}

Table 2. Demographic, Clinical, and Treatment Characteristics From Case Reports

\begin{tabular}{|c|c|c|c|c|c|c|}
\hline Patient Characteristics & Jacobs et $\mathrm{al}^{13}$ & 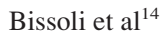 & Inoue et $\mathrm{al}^{15}$ & Inoue et $\mathrm{al}^{15}$ & Nogi et $\mathrm{al}^{16}$ & Cordier et $\mathrm{al}^{23}$ \\
\hline Age & 56 & 71 & 66 & 65 & 48 & 68 \\
\hline Symptom onset latency & & & 23 wk & 26 wk & $4 \mathrm{mo}$ & $2 \mathrm{mo}$ \\
\hline Fever & & + & & + & + & \\
\hline Cough & & + & + & + & + & + \\
\hline Dyspnea & + & & & + & & + \\
\hline Contralateral lung involvement & Yes & No & Yes & Yes & Yes & Yes \\
\hline Biopsy & Yes & Yes & No & Yes & Yes & No \\
\hline Antibiotic treatment & & & & & & Yes \\
\hline Corticosteroid treatment & Yes & Yes & Yes & Yes & Yes & No \\
\hline $\begin{array}{l}\text { Corticosteroid discontinued } \\
\text { relapse }\end{array}$ & No & No & & & & Yes, multiple \\
\hline Outcome & Resolved & Resolved & Improved & Improved & Resolved & $\begin{array}{l}\text { Resolved with long-term steroid } \\
\text { treatment }\end{array}$ \\
\hline Comments & & & & & & $\begin{array}{l}\text { Long-term steroid therapy; } \\
\text { patient carried an ATM gene } \\
\text { mutation }\end{array}$ \\
\hline
\end{tabular}

after completing $50 \mathrm{~Gy}$ radiotherapy to the right breast. She had increased C-reactive protein, Krebs von den Lungen-6, and surfactant protein-D. Chest CT scans and radiography showed patchy infiltrates in all lobes of the right lung. Two weeks later, radiographic consolidation developed in the left lung. After treatment with $30 \mathrm{mg}$ systemic prednisolone, consolidation in the lung fields disappeared, and the patient's symptoms improved. These authors also described a 65-year-old woman who had fever, cough, and shortness of breath 26 weeks after breast cancer radiation. Chest imaging showed contralateral consolidation. She was treated with prednisone for BOOP, which resulted in resolution of symptoms and radiographic consolidation.

Nogi et al ${ }^{16}$ described a 48 -y-old woman with left breast cancer who received $50 \mathrm{~Gy}$ in 25 fractions to the whole breast and a boost of $10 \mathrm{~Gy}$ in 5 fractions to the tumor bed. She developed cough with sputum production 4 months after her radiotherapy was completed. Chest radiography revealed a patchy infiltrate in her left upper lobe. The chest CT showed an air bronchogram in the left upper lung consolidation and new ground-glass opacities in the left lower lung and right upper lung. She was treated with oral levofloxacin for bacterial pneumonia for 2 weeks. Four weeks after completion of the antibiotic therapy, the chest CT revealed the resolution of the original consolidation and ground-glass patchy infiltrates, but new ground-glass patchy infiltrates in the right upper lobe were observed. Lung biopsy showed intra-alveolar organizing pneumonia. Symptoms resolved after 8 months, and her chest CT showed residual fibrosis in the area of the radiated lung. Later, she began taking an herbal medicine containing Scutellaria root for menopausal symptoms. Two weeks after starting the herbal therapy, the respiratory symptoms recurred, and her chest CT showed new ground-glass patchy infiltrates in the right upper lung. She stopped taking the herbal medicine, and both her symptoms and the chest infiltrates resolved. This was likely BOOP from the radiotherapy, although the herb may have been causal.

Cordier et $\mathrm{al}^{17}$ performed genetic testing in a 68 -y-old woman who had invasive left breast ductal carcinoma that was treated with lumpectomy and radiation therapy. Two months after radiation was completed, she developed bilateral ground-glass patchy infiltrates with a reversed halo sign and pleural-based triangle infiltrates with air bronchograms. She received corticosteroid therapy resulting in resolution of the pulmonary infiltrates; however, she had a relapse at $5 \mathrm{mg}$ per day, which was treated successfully with $30 \mathrm{mg}$ prednisone. Her corticosteroid was discontinued after slightly less than 1 year. Three years later, she developed shortness of breath with exertion, cough, fatigue, and abnormal chest radiography. She was treated with prednisone, and her opacities cleared. She had another relapse while taking $15 \mathrm{mg}$ prednisone per day, and eventually she was decreased to $10 \mathrm{mg}$ per day, which was maintained in the long term.

This patient had a family history of ataxia-telangiectasia, and 2 of her 3 children had this disease. Her son had cerebellar syndrome, $\mathrm{T}$ cell prolymphocytic leukemia at age 29 , and he died several months later. Her daughter had cerebellar syndrome, thyroid carcinoma at 22 years of age, and died at 30 years from inhalation pneumonitis and Pseudomonas septicemia. Her daughter's diagnosis was confirmed by the presence of the ataxia telangiectasia mutated (ATM) gene.

The patient with breast cancer had the ATM c.2734C $>T$; p.Gln 912 mutation gene, and the father of the affected children had the ATM c.8494C>T;p.Arg2832Cys mutation. 


\section{Post-Breast CANCER RAdiotherapy AND BOOP}

Women with the ATM mutation have a higher risk of breast cancer, and patients with ataxia telangiectasia are unusually sensitive to therapeutic doses of radiation. ${ }^{18-21}$ Cordier et $\mathrm{al}^{17}$ speculated that this patient may have developed BOOP due to the radio-sensitivity associated with the ATM gene mutation.

This review reports an update of the author's (EK) personal experience with breast cancer postradiotherapy BOOP. ${ }^{1}$ She was a 51 -y-old woman who had ductal carcinoma in situ, and the breast cancer (BRCA) susceptibility gene test was negative. Three hours after the second radiotherapy treatment, she had sudden onset of severe dizziness and lethargy that persisted for $2 \mathrm{~d}$. The radiotherapy treatments were continued, although she continued to have weakness and fatigue. At mid-treatment, she reported dyspnea on exertion. Six months after completion of radiotherapy, the weakness and fatigue worsened. A rheumatological consultation showed the chronic fatigue syndrome, also known as myalgic encephalomyelitis, which was presumably triggered by radiation therapy.

She was diagnosed with severe unilateral BOOP 10 months after completing radiation therapy. She was tested for the ataxia-telangiectasia gene with radio-sensitivity colony survival assays by using fibroblasts with increasing radiation exposure doses of $0,1,2$, and 5 Gray. Two tests were normal, and the third showed slight radio-sensitivity, which was not diagnostic of ataxia-telangiectasia. Nuclear lysates from the fibroblasts tested with Western blot analysis to evaluate the intra-nuclear level of ATM protein were within normal limits.

Corticosteroid treatment at her initial diagnosis was successful, but she had a recurrence with bilateral patchy infiltrates treated with corticosteroids and azathioprine with no additional recurrences. During this course of treatment, her fatigue improved significantly, and when the azathioprine and prednisone were discontinued, her fatigue and debilitation became extremely severe. The rheumatologist speculated that the weakness and fatigue were caused by a "hyperactive" immune system and recommended immunosuppressive therapy. Her fatigue and weakness improved, and she was maintained on azathioprine for 3 years. During this time, she temporarily discontinued azathioprine twice due to infection; both times her fatigue and malaise worsened to the point of debilitation. Her symptoms improved after she had resumed the azathioprine. Standard tests for autoimmune disorders remained negative.

After several years of azathioprine therapy, the myalgic encephalomyelitis symptoms increased significantly; the symptoms responded to single-therapy mycophenolate for 6 months. Prednisone provided some relief, but ultimately methotrexate was needed to diminish weakness, debilitation, and pain. The methotrexate was discontinued because of gastrointestinal symptoms. Tofacitinib was given with improved symptoms after 6 weeks. There has been no recurrence of BOOP for 8 years.

\section{Discussion}

Reports of breast cancer radiotherapy BOOP continue to appear in the medical literature. These new reports have been consistent with prior reports and indicate cigarette smoking, increasing age, central lung distance, and immune factors as risk factors. ${ }^{9,10,22}$

Murofushi et $\mathrm{al}^{9}$ reported $1.4 \%$ incidence of breast-cancer postradiotherapy BOOP, which was similar to other Japanese epidemiological reports. None of these subjects were followed for more than a year, resulting in an underestimate of the incidence because BOOP may appear $>12$ months after radiotherapy. ${ }^{8}$ Sato et al ${ }^{10}$ reported the same $1.4 \%$ incidence. These epidemiological studies confirm that BOOP is a potential complication of breast radiotherapy regardless of age, stage of breast cancer, radiated area, radiation delivery method, and schedule.

Genetic aspects may shed light on the development of $\mathrm{BOOP}$ as it relates to ATM gene and immune dysfunction. Ataxia-telangiectasia is an autosomal recessive disorder characterized by radiosensitivity and an increased cancer risk. ${ }^{18-21}$ The ATM gene is a key regulator of cellular responses to DNA damage caused by radiation. ${ }^{19}$ The description of BOOP occurring in a patient who carried an ATM mutation showed that BOOP may be related to this genetic mutation. ${ }^{17,23}$ In addition, the subjects described in the 2014 report $^{1}$ tested negative for the ATM mutation. It may be prudent for patients with breast cancer contemplating radiotherapy to be questioned about their family history for $A T M$-related disorders because ATM increases the risk of breast cancer, ${ }^{18-21}$ and genetic testing for this mutation can be used as a guide for radiotherapy treatment.

In the subjects reported in our previous review, ${ }^{1}$ breast cancer radiotherapy may have triggered the myalgic encephalomyelitis that developed early in the treatment and persists 9 years later. ${ }^{1}$ Patients with myalgic encephalomyelitis may have immune dysfunction, and poor NK cell cytotoxicity function has correlated with illness severity, ${ }^{24}$ which may explain the improvement with prednisone, azathioprine, methotrexate, and tofacitinib in this patient. In addition, the development of BOOP may be an immune-based inflammatory response caused by a cascade of cytokine events including macrophage and lymphocyte activation with the T1 response. ${ }^{4}$ Epler et $\mathrm{al}^{3}$ reported that patients with BOOP who had a connective tissue disease had a poorer prognosis compared to patients with idiopathic BOOP. Crestani et $\mathrm{al}^{6}$ postulated that radiotherapy of the breast primes or triggers BOOP; and patients with immune dysfunction may be especially susceptible hosts. ${ }^{7}$ Breast radiotherapy 


\section{Post-Breast CANCER RAdiotherapy AND BOOP}

is considered to be relatively contraindicated in patients who have active connective tissue disease involving the skin, such as scleroderma and lupus erythematosus, ${ }^{25}$ because these patients may experience severe toxicity from radiotherapy.

The chest CT scan is vital for determining whether the pulmonary abnormalities are ground-glass inflammation associated with BOOP or correspond to the radiation port as fibrosis associated with radiation pneumonitis. ${ }^{22} \mathrm{We}$ reiterate our recommendation that a chest CT scan be obtained in patients receiving breast cancer radiation who have an abnormal chest radiograph. ${ }^{1}$

Regarding treatment, in the appropriate clinical setting, consideration should be given to actively monitoring women over time with worsening symptoms and chest imaging studies. For example, Kawakami et $\mathrm{al}^{12}$ showed that women with post-breast cancer radiation BOOP may resolve during active monitoring with no prednisone treatment, and concluded that steroids should only be introduced upon onset of hypoxemia, respiratory failure, or unbearable symptoms. Otani et $\mathrm{al}^{22}$ concurred with this approach by stating that treatment should be symptomoriented with no medication necessary for asymptomatic patients, and furthermore, added that relapse of radiation BOOP is common among women receiving steroid therapy.

Radiation pneumonitis is a distinctly different process than BOOP. First, BOOP involves an inflammatory process, whereas fibrosis is found in radiation pneumonitis, and the area of pneumonitis corresponds with the borders of the radiation port. ${ }^{28}$ The clinical and radiological findings between radiation pneumonitis and BOOP may be similar during the early stage; however, radiation pneumonitis becomes fibrotic in the radiation field, whereas BOOP remains inflammatory ground-glass and often migrates from one lung location to another. ${ }^{6}$ BOOP differs in other ways, such as ground-glass opacities outside the radiation field, triangular pleural-based opacities, and the reversed halo sign. As many as one quarter of patients may have both radiation pneumonitis and BOOP. ${ }^{1}$ Although radiation pneumonitis results in permanent fibrosis, this is limited to the radiation site and may not cause clinically important pulmonary impairment, whereas BOOP involves inflammation and resolves completely if successfully treated. ${ }^{5}$ Although chest CT scans may clarify distinctions among radiation pneumonitis, BOOP, or a metastatic process, in rare situations, biopsy remains the accepted standard of diagnosis.

According to the current Common Terminology Criteria for Adverse Events (CTCAE) toxicity scale, BOOP would be classified as pneumonitis, even though BOOP differs from classic radiation pneumonitis in several important ways, such as timing of symptoms, diagnostic work-up, inflammation instead of fibrosis, treatment duration, and prognosis. ${ }^{27}$ The implications of coding BOOP as pneumonitis in the radiotherapy research setting are significant and can lead to misinformation. BOOP is clinically and qualitatively distinct from radiation pneumonitis, and failure to recognize this may lead to delayed diagnosis, misdiagnosis, and misguided treatment, which may compromise patient outcomes.

\section{Conclusion}

BOOP is a distinct and potentially life-threatening disease that can be caused or triggered by many drugs and radiation therapy. We believe BOOP merits inclusion as a separate category in the CTCAE toxicity scale, similar to the addition of the rare but serious retinoic acid syndrome. ${ }^{26} \mathrm{We}$ are not alone in finding the CTCAE pneumonitis category to be problematic. Omarini et $\mathrm{al}^{28}$ suggests that pneumonitis be reclassified by etiology and grade, primarily because pneumonitis caused by targeted therapy is qualitatively different than pneumonitis caused by chemotherapy. Including BOOP in the CTCAE is especially appropriate as newer targeted therapies are developed, tested, and approved for breast cancer and other malignancies.

\section{REFERENCES}

1. Epler GR, Kelly EM. Systematic review of post-radiotherapy bronchiolitis obliterans organizing pneumonia in women with breast cancer. Oncologist 2014;19(12):1216-1226.

2. NIH Consensus Conference. Treatment of early-stage breast cancer. JAMA 1991;265:391-395.

3. Epler GR, Colby TV, McLoud TC, Carrington CB, Gaensler EA. Bronchiolitis obliterans organizing pneumonia. N Engl J Med 1985; 312(3): 152-158.

4. Epler GR. Bronchiolitis obliterans organizing pneumonia, 25 years: a variety of causes, but what are the treatment options? Expert Rev Respir Med 2011;5(3):353-361.

5. American Thoracic Society, European Respiratory Society. American Thoracic Society/European Respiratory Society international multidisciplinary consensus classification of the idiopathic interstitial pneumonias. Am J Respir Crit Care Med 2002;165:277-304.

6. Crestani B, Kambouchner M, Soler P, Crequit J, Brauner M, Battesti JP, Valeyre D. Migratory bronchiolitis obliterans organizing pneumonia after unilateral radiation therapy for beast carcinoma. Eur Respir J 1995;8(2):318-321.

7. Bayle JY, Nesme P, Béjui-Thivolet F, Loire R, Guérin JC, Cordier JF. Migratory organizing pneumonitis "primed" by radiation therapy. Eur Respir J 1995;8(2):322-326.

8. Crestani B, Valeyre D, Roden S, Wallaert B, Dalphin J-C, Cordier J-F. Bronchiolitis obliterans organizing pneumonia syndrome primed by radiation therapy to the breast. Am J Respir Crit Care Med 1998;158 (6):1929-1935.

9. Murofushi KN, Oguchi M, Gosho M, Kozuka T, Sakurai H. Radiation-induced bronchiolitis obliterans organizing pneumonia (BOOP) syndrome in breast cancer patients is associated with age. Radiat Oncol 2015;10(1).

10. Sato H, Ebi J, Tamaki T, Yukawa A, Nakajima M, Ohtake T, Suzuki Y. Incidence of organizing pneumonia after whole-breast radiotherapy 


\section{Post-BREAST CANCER RADIOTHERAPY AND BOOP}

for breast cancer, and risk factor analysis. J Radiat Res 2018;59 (3):298-302.

11. Kong FM, Klein EE, Bradley JD, Mansur DB, Taylor ME, Perez CA, et al. The impact of central lung distance, maximal heart distance, and radiation technique on the volumetric dose of the lung and heart for intact breast radiation. Int J Radiat Oncol Biol Phys 2002;54(3):963971.

12. Kawakami H, Miyabayashi T, Tsubata C, Ota K, Ishida T, Kobayashi O. Spontaneous resolution of thoracic radiation therapy-induced organizing pneumonia: a case series. Respir Med Case Rep 2019; 26:180-184.

13. Jacobs C, Slade M, Lavery B. Doxorubicin and BOOP: a possible near fatal association. Clin Oncol (R Coll Radiol) 2002;14(3):262, letter.

14. Bissoli L, Di Francesco V, Valbusa F, Zivelonghi A, Fantin F, Zamboni M. A case of bronchiolitis obliterans organizing pneumoniae (BOOP) after nine months post-operative irradiation for breast cancer. Age Ageing 2008;37(2):235.

15. Inoue S, Nishiwaki M, Fukuzaki K, Abe S, Shibata Y, Kubota I. Two cases of bronchiolitis obliterans organizing pneumonia syndrome after postoperative irradiation for breast cancer. Excli J 2011;10:246-251.

16. Nogi S, Nakayama H, Tajima Y, Okubo M, Mikami R, Sugahara S, et al. Cryptogenic organizing pneumonia associated with radiation: a report of two cases. Oncol Lett 2014;7(2):321-324.

17. Cordier J-F, Cottin V, Lazor R, Stoppa-Lyonnet D. Monoallelic germline ATM mutation and organizing pneumonia induced by radiation therapy to the breast. Eur Respir J 2016;47(3):997-1000.

18. Geoffroy-Perez B, Janin N, Ossian K, Lauge A, Stoppa-Lyonnet D, Andrieu N. Variation in breast cancer risk of heterozygotes for ataxiatelangiectasia according to environmental factors. Int $\mathrm{J}$ Cancer 2002;99(4):619-623.

19. Bernstein JL, Haile RW, Stovall M, Boice JDJr, Shore RE, Langholz $\mathrm{B}$, et al. Radiation exposure, the ATM gene, and contralateral breast cancer in the Women's Environmental Cancer and Radiation Epidemiology Study. J Natl Cancer Inst 2010;102(7):475-483.

20. Tung N, Lin NU, Kidd J, Allen BA, Singh N, Wenstrup RJ. Frequency of germline mutations in 25 cancer susceptibility genes in a sequential series of patients with breast cancer. J Clin Oncol 2016;34(13):14601468.

21. Easton DF, Pharoah PDP, Antoniou AC, Tischkowitz M, Tavtigian $\mathrm{SV}$, Nathanson KL, et al. Gene-panel sequencing and the prediction of breast-cancer risk. N Engl J Med 2015;372(23):2243-2257.

22. Otani K, Seo Y, Ogawa K. Radiation-induced organizing pneumonia: a characteristic disease that requires symptom-oriented management. Int J Mol Sci 2017;18(2):E281.

23. Cordier J-F, Cottin M, Lazor R, Thivolet-Béjui F. Many faces of bronchiolitis and organizing pneumonia. Semin Respir Crit Care Med 2016;37(3):421-440.

24. Committee on the Diagnostic Criteria for Myalgic Encephalomyelitis/ Chronic Fatigue Syndrome. Beyond Myalgic Encephalomyelitis/ Chronic Fatigue Syndrome: Redefining an Illness, Report Guide for Clinicians. Washington, DC: National Academy of Sciences. Available at: https://www.nap.edu/read/19012/chapter/1. Accessed November 11, 2019.

25. National Comprehensive Cancer Network. NCCN Clinical Practice Guidelines in Oncology: Breast Cancer. Fort Washington, PA: National Comprehensive Cancer Network; 2019: 43.

26. Diederich S. Chest CT for suspected pulmonary complications of oncologic therapies: how I review and report. Cancer Imaging 2016; $16: 7$.

27. U.S. Department of Health and Human Services. Common terminology criteria for adverse events (CTCAE). 2017:131-141.

28. Omarini C, Thanopoulou E, Johnston S. Pneumonitis and pulmonary fibrosis associated with breast cancer treatments. Breast Cancer Res Treat 2014;146(2):245-258 